
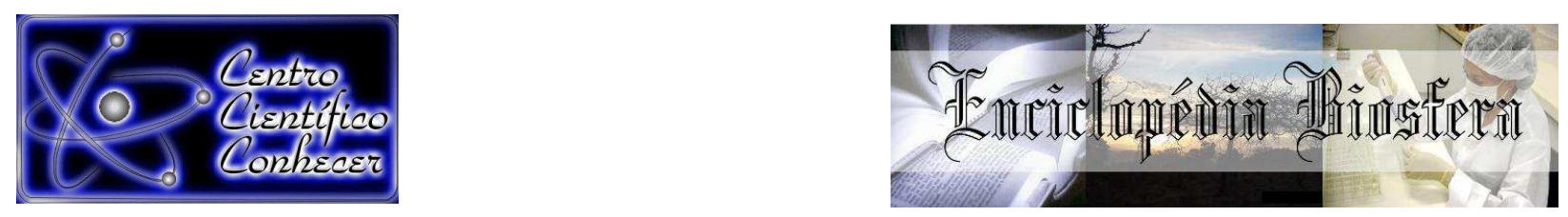

\title{
PRODUTIVIDADE DE CULTIVARES DE SOJA EM SEQUEIRO NO MUNICÍPIO DE PERDIZES, MG
}

\footnotetext{
Fredson de Sousa Correa ; Fausto Antônio Domingos Júnior²; José Carlos Mazetto Júnior ${ }^{3}$; Danyllo Denner de Almeida Costa ${ }^{4}$; José Luiz Rodrigues Torres ${ }^{5}$

1. Engenheiro Agrônomo graduado pela Faculdades Associadas de Uberaba Uberaba-MG; E-mail: fredson.sousacorrea@gmail.com

2. Engenheiro Agrônomo, Professor do Instituto Federal do Triângulo Mineiro (FTM) Campus Uberaba-MG, doutorando do Programa de Pós-Graduação em Agronomia do Instituto de Ciências Agrárias (ICIAG) da Universidade Federal de Uberlândia (UFU);

3. Engenheiro Agrônomo, mestrando do Programa de Pós-Graduação em Agronomia do ICIAG da UFU;

4. Engenheiro Agrônomo, mestrando do Programa de Pós-Graduação em Agronomia do ICIAG da UFU;

5. Professor Titular Doutor em Agronomia - Produção Vegetal do IFTM Campus Uberaba-MG.

Recebido em: 08/04/2017 - Aprovado em: 10/06/2017 - Publicado em: 20/06/2017 DOI: 10.18677/EnciBio_2017A85
}

\begin{abstract}
A necessidade do setor em aumentar a eficiência produtiva e reduzir custos de produção para maior competitividade tem exigido o desenvolvimento de novas tecnologias e novas cultivares. Neste estudo avaliou-se a umidade dos grãos na colheita e a produtividade de cultivares de soja em condição de sequeiro no município de Perdizes, MG. O trabalho foi desenvolvido no período de novembro de 2014 a março de 2015. O delineamento experimental utilizado foi em blocos casualizados (DBC) sendo 23 tratamentos com 12 blocos, constituindo 276 unidades experimentais. A semeadura foi realizada no dia 03 de novembro de 2014 . A cultivar M6210 IPRO foi a que apresentou maior média de produtividade $(3.323 \mathrm{~kg} / \mathrm{ha}$ ) entre todas as cultivares testadas, que foi $22 \%$ superior em comparação à pior média, obtida pela cultivar 95R51 (1.776 kg/ha). As cultivares M6210 IPRO (3.323 kg/ha), AS3730 IPRO (3.278 kg/ha), 5D6215 IPRO (3.209 kg/ha), DESAFIO RR (3.169 $\mathrm{kg} / \mathrm{ha})$, NID7738 IPRO (3.158 kg/ha), M7110 IPRO (3.113 kg/ha) podem ser recomendadas para o cultivo na região de Perdizes, $M G$.
\end{abstract}

PALAVRAS-CHAVE: Eficiência, Produção de soja, Semeadura. 


\title{
VARIETY OF CULTIVARS OF SOY IN RAINFED IN PERDIZES, MG.
}

\begin{abstract}
The necessity of the sector to increase the productive efficiency and reduce the production costs for better competitiveness has required the development of new technologies and new varieties. This study aimed to evaluate the grains moisture at harvest and the productivity of soybean varieties under rainfed conditions in Perdizes, MG. The experiment was conducted from November 2014 to March 2015. The experimental design adopted was the randomized block (RBD) with 23 treatments and 12 blocks, constituting 276 experimental units. The sowing was carried out on 03 November 2014. The variety M6210 IPRO had the highest productivity mean $(3,323 \mathrm{~kg} / \mathrm{ha})$ among all of the varieties tested, $22 \%$ superior than the lowest mean, obtained by the variety 95R51 (1.776 kg/ha). The varieties M6210 IPRO (3.323 kg/ha), AS3730 IPRO (3.278 kg/ha), 5D6215 IPRO (3.209 kg/ha), DESAFIO RR (3.169 kg/ha), NID7738 IPRO (3.158 kg/ha) and M7110 IPRO (3.113 $\mathrm{kg} / \mathrm{ha}$ ) can be recommended for cultivation in Perdizes - MG.
\end{abstract}

KEYWORDS: Efficiency, Soybean production, Sowing.

\section{INTRODUÇÃO}

A elevada produção anual de grãos é um dos grandes impulsionadores da economia do país, em especial a soja (Glycine max (L.) Merrill), que é a cultura mais cultivada em todo território nacional, com uma área estimada em 33,9 milhões de hectares, que está concentrada principalmente na região Centro-Sul do país, totalizando uma produção de 107,6 milhões de toneladas por ano e uma produtividade média de $3,176 \mathrm{~kg} / \mathrm{ha}$ na safra 2016/17 (CONAB, 2017). Dentre os fatores que a tornam uma cultura de tal importância está o teor de proteínas de excelente qualidade (em torno de $40 \%$ ), tanto para alimentação humana quanto para alimentação animal (HIRAKURI \& LAZZAROTTO, 2014).

Em consequência dessa representatividade, a soja está sempre em evidência no quesito pesquisa e inovação, acompanhando um mercado que objetiva explorar ao máximo o potencial produtivo da cultura, sejam por fatores externos (manejo e tratos culturais), sejam por fatores intrínsecos da planta, em especial suas características genéticas. De acordo com ROCHA et al. (2012), o lançamento de cultivares com alta adaptabilidade às condições edafoclimáticas dos trópicos impulsionou a expansão da cultura sob condições de baixa latitude, onde hoje se situam grandes regiões produtoras.

O desenvolvimento de novas cultivares tem sido uma das tecnologias que mais tem contribuído para os aumentos de produtividade e estabilidade de produção, sem custos adicionais ao agricultor (ALMEIDA et al., 1999), entretanto, é fundamental que as cultivares sejam avaliadas por região produtora, pois os genótipos desenvolvidos podem ser promissores em algumas regiões e inviável em outras.

Algumas análises de adaptabilidade e estabilidade feitas na planta no campo permitem identificar as cultivares com comportamento e desenvolvimento mais estáveis e que respondem previsivelmente às variações ambientais, reiterando a especificidade de cada ambiente de cultivo influenciando positiva ou negativamente nas características agronômicas de qualquer cultura (SILVA \& DUARTE, 2006).

A melhor alternativa para determinação da cultivar de melhor desempenho por região é a realização de testes com o maior número possível de cultivares e compará-las conforme suas características produtivas. REZENDE \& CARVALHO 
(2007) recomendaram cultivares de excelentes produtividades para o sul do estado de Minas Gerais, a partir de resultados obtidos que variaram entre 3.049 e 4.395 $\mathrm{kg} / \mathrm{ha}$ de grãos nas cultivares de soja avaliada.

Diante do exposto, neste estudo avaliou-se a umidade dos grãos na colheita e a produtividade de 23 cultivares de soja em condição de sequeiro no município de Perdizes, MG, buscando estabelecer recomendações dos genótipos de melhor desempenho na região.

\section{MATERIAL E MÉTODOS}

O experimento foi realizado em uma área da Fazenda Lagoa da Capa no município de Perdizes, MG. Localizada a 19²7'17,64" de latitude sul e 47²2'05,75" de longitude oeste, com altitude de $1084 \mathrm{~m}$, em uma área de reforma de cana-deaçúcar.

O solo da área foi classificado como Latossolo Vermelho distrófico de textura média (EMBRAPA, 2013), apresentando na camada de 0,0-0,40 m: $\mathrm{pH} \mathrm{CaCl}^{2}$ 5,2; 49 $\mathrm{mg} / \mathrm{dm}^{3}$ de $\mathrm{P}$ (resina); 3 mmol $/ \mathrm{dm}^{3}$ de $\mathrm{K}^{+} ; 26 \mathrm{mmol}_{\mathrm{d}} / \mathrm{dm}^{3} \mathrm{de} \mathrm{Ca}^{2+} ; 10 \mathrm{mmol} / \mathrm{dm}^{3} \mathrm{de}$ $\mathrm{Mg}^{2+} ; 46 \mathrm{mmol}_{\mathcal{L}} / \mathrm{dm}^{3}$ de H+Al, $44 \mathrm{mmol}_{d} / \mathrm{dm}^{3} \mathrm{SB}, 90 \mathrm{mmol}_{c} / \mathrm{dm}^{3}$ de T, V\% de 49 e 31 $\mathrm{g} / \mathrm{dm}^{3}$ de matéria orgânica.

O clima da região, segundo classificação de Koppen, é Aw, tropical quente úmido com inverno frio e seco, com precipitação e temperatura média no período do experimento de $1190 \mathrm{~mm}$ e $24,98^{\circ} \mathrm{C}$, segundo dados obtidos pelas estações climatológicas INMET-EPAMIG. No período em que o estudo foi conduzido ocorreu um veranico que causou um déficit hídrico no estádio reprodutivo da cultura, no qual interferiu nas médias das produtividades (Figura 1).

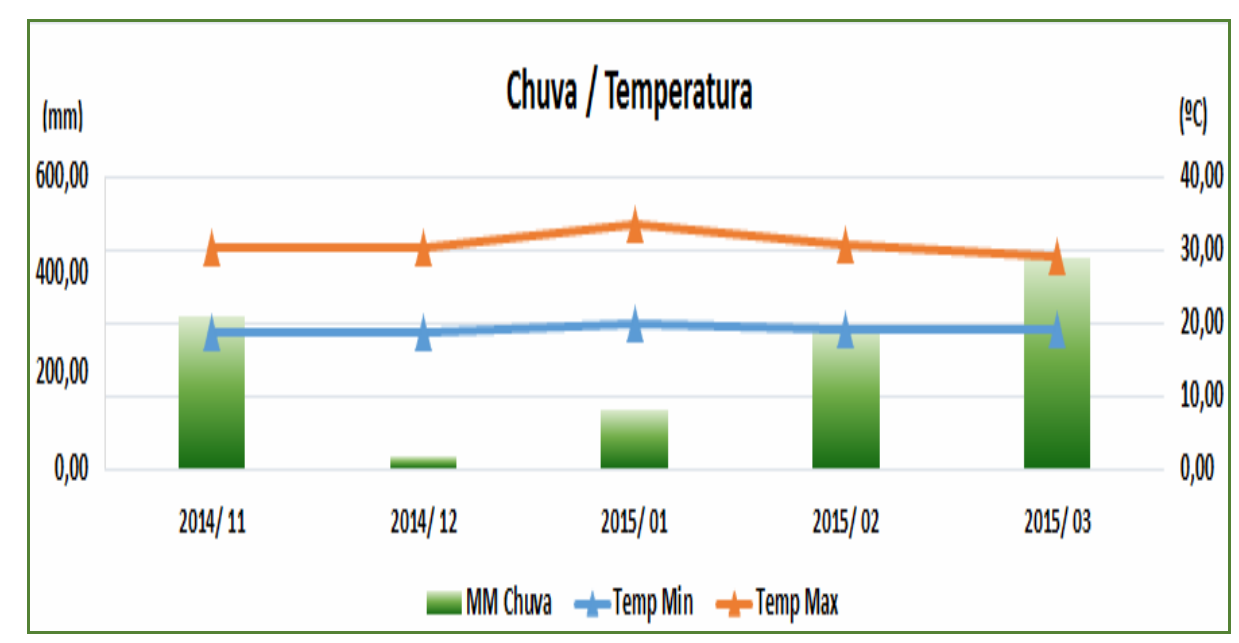

FIGURA 1. Precipitação pluviométrica e temperaturas máximas e mínimas no período de 11/2014 a 03/2015 da área experimental.

O delineamento experimental utilizado foi de blocos casualizados (DBC) e foi avaliado 23 cultivares (tratamentos) (Tabela 1), com 12 blocos (repetições), que totalizaram 276 unidades experimentais, espaçadas de $0,50 \mathrm{~m}$ por $5,0 \mathrm{~m}$ de comprimento, em uma área total de $55.200 \mathrm{~m}^{2}$, foram avaliadas as quatro linhas centrais, sendo deixadas quatro linhas de cada lado de bordadura. 
TABELA 1. Descrição cultivares, grupo de maturação, tipo de crescimento e população utilizados no experimento.

\begin{tabular}{cccc}
\hline Cultivares & Maturação & Crescimento & População \\
\hline M6210 IPRO & 6.3 & Indeterminado & 320.000 \\
AS3730 IPRO & 7.4 & Determinado & 320.000 \\
5D6215 IPRO & 7.1 & Indeterminado & 320000 \\
DESAFIO RR & 7.1 & Indeterminado & 360.000 \\
NS7338 IPRO & 6.4 & Indeterminado & 400.000 \\
M7110 IPRO & 7.0 & Indeterminado & 360.000 \\
ANTA 82RR & 6.2 & Indeterminado & 440.000 \\
INOX7062 TMG & 5.5 & Indeterminado & 240.000 \\
M6410 IPRO & 6.1 & Indeterminado & 320.000 \\
PONTA IPRO & 6.3 & Indeterminado & 360.000 \\
1364A38 IPRO & 6.8 & Indeterminado & 320.000 \\
NS7209 IPRO & 7.3 & Indeterminado & 360.000 \\
5D7315 IPRO & 6.2 & Indeterminado & 320.000 \\
5D634 RR DOW & 6.4 & Indeterminado & 400.000 \\
97R21 & 7.3 & Indeterminado & 300.000 \\
NS5959 IPRO & 7.4 & Indeterminado & 360.000 \\
5G685 RR DOW & 7.3 & Indeterminado & 320.000 \\
5D555 RR DOW & 7.2 & Indeterminado & 300000 \\
SYNG 1163 & 5.2 & Indeterminado & 360.000 \\
97Y07 & 5.5 & Indeterminado & 300.000 \\
5D615 RR DOW & 7.0 & Indeterminado & 400.000 \\
95Y21 & 7.2 & Indeterminado & 360.000 \\
95R51 & 5.9 & Indeterminado & 400.000 \\
\hline
\end{tabular}

No preparo do solo foram utilizados: o eliminador de soqueira, grade aradora, distribuidor de calcário, subsolador, grade intermediária e grade niveladora no preparo do solo. Logo após o uso de eliminador de soqueiras foi feito a calagem, para elevação da saturação de bases do solo para $60 \%$, o que levou a aplicação de 2 ton/ha de calcário dolomítico $(16 \% \mathrm{MgO})$ e PRNT de 90\%. Este calcário foi incorporado a $0,40 \mathrm{~m}$ de profundidades pelos equipamentos utilizados na sequencia de preparo do solo.

As sementes foram tratadas com uma mistura de fungicidas (Piraclostrobina + Tiofanato metílico e Fipronil) a $200 \mathrm{~mL}$ por $100 \mathrm{~kg}$ de sementes. A população de cada cultivar do experimento foi definida de acordo com a especificação de cada empresa para região.

O plantio foi realizado no dia 03 de novembro de 2014, com o auxilio de um trator Valtra BT190 e com uma plantadeira PST2 Ultraflex Tatu de 12 linhas. Foi utilizado um pulverizador terrestre Uniporte 3030 Jacto para as operações de tratos culturais que a cultura da soja necessita para seu pleno desenvolvimento.

A adubação realizada em pré-plantio foi aplicado a lanço na dose de $220 \mathrm{~kg} / \mathrm{ha}$ do formulado $00-00-54+1 \%$ B. Em plantio foram aplicados $280 \mathrm{~kg} / \mathrm{ha}$ da formulação 8-40-10 aplicados no sulco de plantio, realizando juntamente a semeadura, no mesmo sulco de plantio, a 0,05 $\mathrm{m}$ de profundidade, abaixo e ao lado das sementes de soja.

Para o controle de plantas daninhas, utilizou-se o herbicida Glifosate Potássico (2 l/ha) e para o controle de pragas, inseticidas a base de Profenofós + Lufenuron (0,3 I/ha) e Tiametoxam + Lambda-Cialotrina (0,2 I/ha). Para o controle de doenças, foi realizado com fungicida com o princípio ativo Trifloxistrobina + Protioconazol (0,4 I/ha) e Azoxistrobina + Ciproconazol (0,35 I/ha). As dosagens, 
produtos e épocas de aplicação dos insumos, foram realizados de acordo com as recomendações da EMBRAPA (1999).

As parcelas experimentais foram colhidas manualmente e trilhadas em uma trilhadora de experimentos da empresa SB Máquinas. A seguir, os grãos foram levados para a estação de pesquisa para pesagem e determinação de umidade. Para determinar umidade, utilizou-se um medidor do tipo Gehaka modelo G 810 e balanças de precisão Filizola. A normalidade dos dados foi verificada pelo teste de Shapiro - Wilk, a 0,05 de probabilidade, submetidos à análise de variância e as médias comparadas pelo teste de Scott-Knott ao nível de 5\% de probabilidade.

\section{RESULTADOS E DISCUSSÃO}

A umidade do grão no momento da colheita é uma variável importante a ser analisada em várias culturas, na soja, a colheita deve ser iniciada logo que o teor de umidade dos grãos atingir valores variando entre 13 e $15 \%$, para que se minimize os problemas de danos mecânicos e perdas na colheita (SILVEIRA \& CONTE, 2013).

Neste estudo, as cultivares que estavam dentro desta faixa de umidade, em ordem decrescente foi a 95Y21 com 15,03\%, seguida pela 97R21 (14,93\%), AS3730 IPRO (14,90\%), 97 Y07 (14,79\%), M6210 IPRO (14,73\%), NID7338 IPRO (14,39\%), 5D634 RR DOW (14,18\%), 5G555 RR DOW (14,07\%), 5D615 RR DOW (13,83\%) e NID7209 IPRO $(13,73)$ (Tabela 2).

Sementes colhidas com teor de umidade superior a $15 \%$ estão sujeitas a maior incidência de danos mecânicos latentes (não aparentes), sendo que estes danos podem ocorrer nas cultivares 5D6215 IPRO e SYNG 1163, que apresentaram as maiores porcentagens de umidade, de $20,54 \%$ e $20,73 \%$ respectivamente, diferindo-se estatisticamente das demais.

Alguns outros pesquisadores destacam a umidade máxima de $18 \%$ que os grãos de soja podem estar na hora da colheita. HAMER \& PESKE (1997) afirmam que a colheita da soja deve ser realizada quando o teor de umidade dos grãos estiver entre 14 a 18\%, enquanto que MOHLER (2010) destaca que a umidade ideal varia entre 16 a 18\% para a colheita e de 9 a 12\% para o armazenamento, para períodos de 1 a 5 anos.

TABELA 2. Peso bruto (PB), em $\mathrm{kg}$, umidade (U), em porcentegem, peso líquido (PL), em $\mathrm{kg}$ e produtividade (Prod), em $\mathrm{kg} / \mathrm{ha}$, de grãos com umidade corrigida para $14 \%$ de diferentes cultivares de soja cultivadas em Perdizes, MG.

\begin{tabular}{lcccc}
\hline Cultivares & PB & $\mathbf{U}$ & $\mathbf{P L}$ & Prod \\
\hline M6210 IPRO & $3,35 \mathrm{a}$ & $14,73 \mathrm{e}$ & $3,32 \mathrm{a}$ & $3.323 \mathrm{a}$ \\
AS3730 IPRO & $3,31 \mathrm{a}$ & $14,90 \mathrm{e}$ & $3,28 \mathrm{a}$ & $3.278 \mathrm{a}$ \\
5D6215 IPRO & $3,43 \mathrm{a}$ & $20,54 \mathrm{a}$ & $3,21 \mathrm{a}$ & $3.209 \mathrm{a}$ \\
DESAFIO RR & $3,23 \mathrm{~b}$ & $15,73 \mathrm{~d}$ & $3,17 \mathrm{a}$ & $3.169 \mathrm{a}$ \\
NS7338 IPRO & $3,17 \mathrm{~b}$ & $14,39 \mathrm{e}$ & $3,16 \mathrm{a}$ & $3.158 \mathrm{a}$ \\
M7110 IPRO & $3,22 \mathrm{~b}$ & $17,18 \mathrm{c}$ & $3,11 \mathrm{a}$ & $3.113 \mathrm{a}$ \\
ANTA 82RR & $3,20 \mathrm{~b}$ & $18,00 \mathrm{c}$ & $3,06 \mathrm{~b}$ & $3.063 \mathrm{~b}$ \\
INOX7062 TMG & $3,09 \mathrm{c}$ & $16,14 \mathrm{~d}$ & $3,02 \mathrm{~b}$ & $3.021 \mathrm{~b}$ \\
M6410 IPRO & $3,17 \mathrm{~b}$ & $18,91 \mathrm{~b}$ & $3,01 \mathrm{~b}$ & $3.012 \mathrm{~b}$ \\
PONTA IPRO & $3,10 \mathrm{c}$ & $17,03 \mathrm{c}$ & $3,00 \mathrm{~b}$ & $3.003 \mathrm{~b}$ \\
1364A38 IPRO & $3,02 \mathrm{c}$ & $17,48 \mathrm{c}$ & $2,92 \mathrm{~b}$ & $2.916 \mathrm{~b}$ \\
NS7209 IPRO & $2,90 \mathrm{c}$ & $13,73 \mathrm{e}$ & $2,90 \mathrm{~b}$ & $2.903 \mathrm{~b}$ \\
5D7315 IPRO & $3,00 \mathrm{c}$ & $18,67 \mathrm{~b}$ & $2,86 \mathrm{~b}$ & $2.861 \mathrm{~b}$ \\
5D634 RR DOW & $2,70 \mathrm{~d}$ & $14,18 \mathrm{e}$ & $2,69 \mathrm{c}$ & $2.685 \mathrm{c}$ \\
97R21 & $2,67 \mathrm{~d}$ & $14,93 \mathrm{e}$ & $2,65 \mathrm{c}$ & $2.648 \mathrm{c}$ \\
NS5959 IPRO & $2,64 \mathrm{~d}$ & $6,08 \mathrm{~d}$ & $2,58 \mathrm{c}$ & $2.585 \mathrm{c}$
\end{tabular}




$\begin{array}{lcccc}\text { 5G685 RR DOW } & 2,70 \mathrm{~d} & 19,13 \mathrm{~b} & 2,56 \mathrm{c} & 2.564 \mathrm{c} \\ \text { 5D555 RR DOW } & 2,48 \mathrm{e} & 14,07 \mathrm{e} & 2,48 \mathrm{~d} & 2.476 \mathrm{~d} \\ \text { SYNG 1163 } & 2,65 \mathrm{~d} & 20,73 \mathrm{a} & 2,47 \mathrm{~d} & 2.474 \mathrm{~d} \\ \text { 97Y07 } & 2,50 \mathrm{e} & 14,79 \mathrm{e} & 2,47 \mathrm{~d} & 2.470 \mathrm{~d} \\ \text { 5D615 RR DOW } & 2,46 \mathrm{e} & 13,83 \mathrm{e} & 2,46 \mathrm{~d} & 2.461 \mathrm{~d} \\ \text { 95Y21 } & 1,91 \mathrm{f} & 15,03 \mathrm{e} & 1,89 \mathrm{e} & 1.885 \mathrm{e} \\ \text { 95R51 } & 1,81 \mathrm{f} & 15,55 \mathrm{~d} & 1,78 \mathrm{e} & 1.776 \mathrm{e} \\ \text { CV \% } & 7,99 & 8,54 & 7,98 & 7.98\end{array}$

As médias seguidas pela mesma letra não diferem estatisticamente entre si. Foi aplicado o Teste de Scott-Knott ao nível de $5 \%$ de probabilidade.

Com relação à produtividade da cultura, as cultivares M6210 IPRO, AS3730 IPRO, 5D6215 IPRO, DESAFIO RR, NID7738 IPRO e M7110 IPRO, apresentaram as produtividades de $3.323 ; 3.278 ; 3.209 ; 3.169 ; 3.158$ e $3.113 \mathrm{~kg} / \mathrm{ha}$, respectivamente, que foram superiores e diferiram estatisticamente das demais cultivares avaliadas. Dentre estas cultivares, pode-se destacar que a única cultivar com hábito de crescimento determinado (AS3730 IPRO) apresentou a segunda maior média de produtividade (3.278 kg/ha) (Tabela 2).

As cultivares 95R51 e 95Y21 apresentaram a produtividade de 1.776 e 1.885 $\mathrm{kg} / \mathrm{ha}$ respectivamente, que foram os menores valores, quando comparados às outras cultivares avaliadas. Relacionando umidade dos grãos na colheita e produtividade nas seis cultivares de maior rendimento, apenas as cultivares M6210 IPRO, AS3730 IPRO e NID7338 IPRO apresentaram umidade inferior a 15\%, enquanto que a 5D6215 IPRO, DESAFIO RR e M7110 IPRO apresentaram umidade de $20,54 \%, 15,73 \%$ e $17,18 \%$ respectivamente. Desta forma, nota-se não existir relação entre os valores de umidade obtidos no momento da colheita com os índices de produtividade, obtidos a partir do peso líquido calculado.

A produtividade de uma cultura é definida pela interação entre o genótipo da planta, ambiente de produção e manejo, sendo que para obtenção de altos rendimentos é necessário que as condições ambientais sejam favoráveis em todos os estádios de crescimento da soja (RITCHIE et al., 1994).

Segundo REZENDE \& CARVALHO (2007), na Universidade Federal de Lavras, situada à latitude de $21^{\circ} 14^{\prime} \mathrm{S}$, longitude $45^{\circ} 00 \mathrm{~W}$ e altitude de $918 \mathrm{~m}$, conduzido no ano agrícola 2002/03, também avaliando peso em diferentes cultivares de soja, obtiveram produtividades satisfatórias no cultivo de soja, com destaque para Vencedora (4.395 kg/ha), Paiaguás (3.897 kg/ha), Aventis 2056-7 (3.780 kg/ha), Monarca (3.646 kg/ha) e FT $2000(3.498 \mathrm{~kg} / \mathrm{ha})$. Esses valores são superiores à maior média alcançada. Isso se deve não só às cultivares utilizadas no experimento, mas também à adaptabilidade das mesmas às condições edafoclimáticas da região.

Em Aquidauana, MS, no Cerrado brasileiro, TORRES et al. (2014) avaliaram as cultivares BMX Magna RR, Monsoy 7908 RR, Nidera 7321 RG, BRS 245 RR e Syngenta 3358 RR e concluíram que o genótipo BRS 245 apresentou o maior valor absoluto de produtividade, com uma média aproximada de $3.383,33 \mathrm{~kg} / \mathrm{ha}$, apesar de não haver diferença significativa entre as demais avaliadas. Esta produtividade obtida foi superior quando comparado às médias observadas neste estudo, pois a maior média obtida foi na cultivar M6210 IPRO (3.323 kg/ha), no entanto, com diferença de apenas $65 \mathrm{~kg} / \mathrm{ha}$.

TORRES et al. (2015), em Aquidauana, MS, avaliaram seis cultivares transgénicas de soja (P98Y70, CD 238, CD 241, BRS 255, VMAX e NK 7059). Os autores concluíram que o genótipo P98Y70 foi o mais produtivo com média de 2.348 $\mathrm{kg} / \mathrm{ha}$. Tal média produtiva foi $974 \mathrm{~kg} / \mathrm{ha}$ inferior ao apresentado pela cultivar M6210 
IPRO, maior média obtida no presente trabalho, e $765 \mathrm{~kg} / \mathrm{ha}$ inferior a M7110 IPRO que apresentou a menor média dentre as seis cultivares mais produtivas avaliadas neste trabalho.

\section{CONCLUSÕES}

A cultivar M6210 IPRO foi a que apresentou maior média de produtividade (3.323 $\mathrm{kg} / \mathrm{ha}$ ) entre todas as cultivares testadas, que foi $22 \%$ superior em comparação à pior média, obtida pela cultivar 95R51 (1.776 kg/ha).

As cultivares M6210 IPRO (3.323 kg/ha), AS3730 IPRO (3.278 kg/ha), 5D6215 IPRO (3.209 kg/ha), DESAFIO RR (3.169 kg/ha), NID7738 IPRO (3.158 $\mathrm{kg} / \mathrm{ha})$, M7110 IPRO (3.113 kg/ha) podem ser recomendadas para o cultivo na região de Perdizes, MG.

\section{REFERÊNCIAS}

ALMEIDA, L. A. de; KIIHL, R. A. de S.; MIRANDA, M. A. C. de; CAMPELO, G. J. de A. Melhoramento da soja para regiões de baixa latitude. In: QUEIRÓZ, M. A. de; GOEDERT, C. O.; RAMOS, S. R. R. (Org.). Recursos genéticos e melhoramento de plantas para o Nordeste brasileiro. Brasília: EMBRAPA, 1999. p. 73-88. Disponível em: <https://www.embrapa.br/busca-de-publicacoes/-/publicacao/1538 31/recursos-geneticos-e-melhoramento-de-plantas-para-o-nordeste-brasileiro>.

Acesso em: 24 out. 2016.

CONAB - Acompanhamento da safra brasileira de grãos. Sexto levantamento. Safra 2016/17, v. 4, n. 6, p. 1-176, 2017. Disponível em: <http://www.conab.gov.br/OlalaCMS/uploads/arquivos/17_03_14_15_28_33_boletim _graos_marco_2017bx.pdf>. Acesso em: 03 mar. 2017.

EMBRAPA. Trigo. 2005. Empresa Brasileira de Pesquisa Agropecuária - Centro Nacional de Pesquisa do Trigo. Disponível em: <http://www.cnpt.embrapa.br/sistprod/soja04/tab1.htm>. Acesso em: 25 abr. 2016.

EMBRAPA. Recomendações para a cultura da soja na região central do Brasil 1999/2000. Londrina: EMBRAPA-CNPSo, 1999. 226p.

EMBRAPA. Soja. 2015. Empresa Brasileira de Pesquisa Agropecuária. Disponível em: <https://www.embrapa.br/soja/cultivos/soja1>. Acesso em: 25 abr. 2016.

HAMER, E.; PESKE, S. T. Colheita de sementes de soja com alto teor de umidade: I. Qualidade física. Revista Brasileira de Sementes, v. 19, p. 106-110, 1997.

HIRAKURI, M. H.; LAZZAROTTO, J. J. O agronegócio da soja nos contextos mundial e brasileiro. Documentos Embrapa, Londrina, n. 349, 2014. Disponivel em:

<http://www.infoteca.cnptia.embrapa.br/bitstream/doc/990000/1/Oagronegociodasoj anoscontextosmundialebrasileiro.pdf>. Acesso em: 01 fev. 2017.

REZENDE, P. M.; CARVALHO, E. A. Avaliação de cultivares de soja (Glycine max (L.) Merrill) para o sul de Minas Gerais. Ciência e Agrotecnologia, v. 31, n. 6, p. 
1616-1623, 2007. Disponível em: <http://dx.doi.org/10.1590/S141370542007000600003>. doi: 10.1590/S1413-70542007000600003.

RITCHIE, S. W.; HANWAY, J. J.; THOMPSON, H. E.; BENSON, G. O. How a soybean plant develops. lowa State University of Science and Thechnology, Cooperative Extension Service, 1985. 20p. (Special Report, 53). Disponível em: <http://publications.iowa.gov/14855/1/1985\%20How\%20a\%20Soybean\%20Plant\%20 Develops.pdf>. Acesso em: 30 jan. 2017.

ROCHA, R. S.; DA SILVA, J. A. L.; NEVES, J. A.; SEDIYAMA, T.; DE CÁSSIA TEIXEIRA, R. Desempenho agronômico de variedades e linhagens de soja em condições de baixa latitude em Teresina-PI. Revista Ciência Agronômica, v. 43, n. 1 , p. 154-162, 2012. Disponível em: <http://dx.doi.org/10.1590/S1806$66902012000100019>$. doi: 10.1590/S1806-66902012000100019.

SILVA, W. C. J. e; DUARTE, J. B. Métodos estatísticos para estudo de adaptabilidade e estabilidade fenotípica em soja. Pesquisa Agropecuária Brasileira, v. 41, n. 1, p. 23-30, 2006. Disponível em: <http://dx.doi.org/10.1590/S0100-204X2006000100004>. doi: 10.1590/S0100204X2006000100004.

SILVEIRA, J. M.; CONTE, O. Determinação de perdas na colheita de soja: copo medidor da. Embrapa. Londrina-PR, 2013, 28p. Disponível em: $<$ https://ainfo.cnptia.embrapa.br/digital/bitstream/item/97495/1/Manual-Copo-

Medidor-baixa-completo.pdf>. Acesso em: 05 abr. 2017.

TORRES, F. E.; DAVID, G. V.; TEDORO, P. E.; RIBEIRO, L. P.; CORREA, C. G.; JUNIOR, R. A. L. Desempenho agronómico e dissimilaridade genética entre genótipos de soja. Ciencias Agrárias. Aquidauana, v. 38, n. 1, p.111-117, abril, $2015 . \quad$ Disponível em: <http://www.scielo.mec.pt/scielo.php?script=sci_arttext\&pid=S0871-

018X2015000100016>. Acesso em: 15 abr. 2017.

TORRES, F. E.; SILVA, E. C.; TEODORO, P. E. Desempenho de genótipos de soja nas condições edafoclimáticas do ecótonno Cerrado-Pantanal. Interações. Campo Grande, v. 15, n. 1, p. 71-7, 2014. Disponível em: <http://dx.doi. org/10.1590/S151870122014000100007>. doi: 10.1590/S1518-70122014000100007.

VERDÉLIO. A. Conab revisa previsão e safra 2015/2016 deve chegar a 209 milhões toneladas.

Disponível

em:

<http://agenciabrasil.ebc.com.br/economia/noticia/2016-04/conab-revisa-previsao-esafra-20152016-deve-chegar-209-milhoes-toneladas>. Acesso em: 10 mai. 2016. 